Revista de Psicología Vol. 30 (1), 2012 (ISSN 0254-9247)

\title{
Propiedades psicométricas del Maslach Burnout Inventory-GS en una muestra multiocupacional venezolana
}

\author{
Anthony Constant Millán de Lange ${ }^{1}$ y María Eugenia D’Aubeterre López ${ }^{2}$ \\ Universidad Metropolitana, Caracas, Venezuela
}

La evaluación del estrés laboral es uno de los aspectos de mayor importancia en el ámbito actual de la salud psicológica ocupacional, más aún cuando llega a niveles crónicos de afectación. Se presentan los resultados psicométricos de la validación venezolana de la versión cubana del Maslach Burnout Inventory-General Survey (MBI-GS). Los resultados revelan la adecuada confiabilidad del instrumento y sugieren la necesidad de eliminar el ítem 1 para asegurar la validez de constructo del mismo. También se presentan indicadores que revelan la validez de criterio divergente del MBI-GS sobre el grado de Bienestar Psicológico de los participantes.

Palabras clave: Síndrome de Desgaste Profesional, fiabilidad, validación.

Psychometric properties of the Maslach Burnout Inventory-GS in a Venezuelan sample The assessment of work stress is one of the most important aspects in the current scope of occupational psychological health, especially when it comes to chronic levels of involvement. Here are the results of the Venezuelan psychometric validation of the Cuban version of the Maslach Burnout Inventory-General Survey (MBI-GS). Results show adequate reliability of the instrument and suggest the need to remove item 1 to ensure its construct validity. Some indicators reveal the divergent criterion validity of the MBI-GS on the degree of the participant's psychological well-being.

Key words: Burnout, Burnout Syndrome, reliability, validation.

1 Docente Asociado, Departamento de Ciencias del Comportamiento, Escuela de Psicología. Contacto: Principio Carretera Petare-Guarenas, Campus Universidad Metropolitana, Edificio de Aulas 2, Piso 2, Departamento de Ciencias del Comportamiento, Apartado Postal 76.819, Caracas, Venezuela; anmillan@unimet.edu.ve

2 Docente Asociada, Departamento de Ciencias del Comportamiento, Escuela de Psicología. Contacto: Principio Carretera Petare-Guarenas, Campus Universidad Metropolitana, Edificio de Aulas 2, Piso 2, Departamento de Ciencias del Comportamiento, Apartado Postal 76.819, Caracas, Venezuela; mdaubeterre@unimet.edu.ve 

De acuerdo con Maslach (2009), el burnout es un síndrome psicológico que implica una respuesta prolongada a estresores interpersonales de tipo crónico en el trabajo. Sus dimensiones desde el modelo propuesto por esta autora son tres: agotamiento extenuante, sentimiento de cinismo y desapego por el trabajo, y sensación de ineficacia y falta de logros. El agotamiento representa el componente de estrés individual básico del burnout y se refiere a sentimientos de estar sobreexigido y vacío de recursos emocionales y físicos. Las causas principales del origen de este agotamiento son la sobrecarga laboral y el conflicto personal en el trabajo. El cinismo representa el componente del contexto interpersonal del burnout y se refiere a una respuesta negativa, insensible o excesivamente apática a diversos aspectos del trabajo. La ineficacia representa el componente de autoevaluación del burnout y se refiere a los sentimientos de incompetencia y carencia de logros y productividad en el trabajo. Este sentido disminuido de autoeficacia es exacerbado por una carencia de recursos de trabajo, así como por una falta de apoyo social y de oportunidades para desarrollarse profesionalmente.

Shirom (2009) señala que puede considerarse al burnout como una reacción afectiva a un estrés prolongado en el trabajo, cuyo contenido medular es el agotamiento gradual de los recursos energéticos intrínsecos de los individuos en el transcurso del tiempo, incluyendo la expresión de agotamiento emocional, fatiga física y cansancio cognitivo. El modelo original de medida del síndrome desde la postura de Maslach y Jackson (1981) se desarrolla dentro del ámbito clínicohospitalario, a través del inventario MBI-HSS, en donde a diferencia del inventario de MBI-GS, se denomina a la dimensión de Cinismo como Despersonalización y a la dimensión de Ineficacia como Baja Realización Personal, manteniendo igual la denominación de Agotamiento Emocional. 
En tal sentido, la despersonalización se refería a la respuesta negativa, cínica o excesivamente indiferente hacia otras personas en el trabajo y representaba el componente interpersonal del burnout. La baja realización personal se refería a los sentimientos de declinación en la competencia y productividad de uno y a la sensación de autoeficacia disminuida y representaba el componente de autoevaluación del burnout (Maslach, 2009). De acuerdo con Shirom (2009), la incorporación del Cinismo como etiqueta denominadora de la antigua dimensión de Despersonalización plantea nuevos problemas, ya que el mismo aún tiene que establecer su validez discriminante, por lo que estudios que amplíen el análisis a la validez convergente o divergente de la medida presentarían evidencia empírica de la adecuación de la nueva denominación. En tal sentido, el presente estudio incorporó la medida de Bienestar Psicológico como criterio divergente de la validación latinoamericana del MBI-GS. Por su parte, Gil-Monte (2001) señala que los cambios de denominación en las dimensiones del instrumento MBI-HSS y MBI-GS responden a la necesidad de ampliación del constructo de burnout desde este contexto clínico-hospitalario, a la aproximación generalista del MBI-GS, y ello supone conceptualmente que en el caso del Cinismo, se deja de hacer énfasis directamente en la forma de interacción con las personas a quienes se le presta el servicio (como lo hacía la dimensión de despersonalización), sino que más bien refleja indiferencia o actitudes de distanciamiento hacia el trabajo en general.

Una lectura más acuciosa permite observar que el cambio de denominación en el caso de la despersonalización no supone una anulación de la misma, ya que ella permanece como una aproximación indirecta por efecto de la actitud cínica en el trabajo. Al obrar con indiferencia con respecto a las propias labores del rol (cuando supone el trato con personas) implica una cosificación de las personas a las cuales se le presta un servicio, dado que ellas son el componente principal de la ejecución del rol laboral. Por lo tanto, al manifestarse el cinismo, se expresa en la despersonalización de las relaciones interpersonales con respecto a los que reciben el servicio. 
Con respecto a los instrumentos de medida, además de la diferencia ya señalada entre el MBI-HSS y el MBI-GS, Hernández, Terán, Navarrete y León (2007) reseñan una lista de 14 instrumentos. En un esfuerzo similar, Garcés de los Fayos, López y García (1997) comparan el Burnout Measures de Pines, Aronson y Kafry de 1981, el Staff Burnout Scale de Jones de 1980, y el Maslach Burnout Inventory de Maslach y Jackson de 1981, encontrando diferencias en el número de ítems y la naturaleza de las dimensiones que estos evalúan (de 3 a 4 dimensiones).

Existen casos como el Cuestionario para la Evaluación del Síndrome de Quemarse por el Trabajo en profesionales que trabajan con personas con discapacidad (CESQT-PD) de Gil-Monte (2005), en los que se incorporan hasta 7 dimensiones de evaluación. Otros instrumentos amplían la manifestación del síndrome de burnout únicamente a partir del contexto laboral y lo ubican también dentro de las relaciones maritales (Pines, 1987). Finalmente, Shirom (2009) comenta que la investigación acerca del burnout, independientemente del enfoque conceptual utilizado, en sus inicios se focalizó casi exclusivamente en profesionales orientados a las personas y en empleados del sector público, limitando su capacidad de generalización, ya que en estos contextos la naturaleza del cargo les exige invertir continuamente energía emocional, cognitiva y física en los receptores de sus servicios. Por ello, cualquier propuesta de validación de estos instrumentos debería incorporar también al sector privado de la economía para asegurar la debida heterogeneidad de la muestra, que exige cualquier análisis basado en coeficientes de correlación o covariación (Aron \& Aron, 2001), más aún cuando estos están centrados en la validación de instrumentos (Anastasi \& Urbina, 1998).

Hair, Anderson, Tatham y Black (1999) señalan como tamaño mínimo para estudios que hacen uso de la técnica del análisis factorial exploratorio, al menos 10 participantes por ítem, lo que supondría un tamaño mínimo requerido para esta muestra de 160 participantes. En este mismo orden de ideas, Prieto y Muñiz (2000), haciendo eco del modelo para evaluar la calidad de los tests del Colegio Oficial 
de Psicólogos de España, desarrollado en conjunción con la Asociación de Psicólogos Profesionales (EFPPA) y la Comisión Internacional de Test (ITC), señalan que el uso oportuno de muestras en contextos de validación de constructo es de 200 a 500 participantes como mínimo. Además de esto el baremo considera de importancia la presentación de los resultados de análisis de ítem.

Es por ello que en el presente estudio se busca analizar la validez de constructo de la adaptación latinoamericana del MBI-GS, realizada por Oramas et al. (2007) a partir de la adaptación española que hiciesen Moreno-Jiménez, Carvajal y Escobar (como se cita en Orama et al., 2007) del instrumento original de Maslach y Jackson (1981). Esto se hace considerando una muestra de participantes venezolanos cuyo tamaño está dentro de los límites mínimos establecidos por el criterio de potencia estadística para la técnica del análisis factorial (al menos 160 participantes) y que cuenta con la debida heterogeneidad en función a los sectores ocupacional (público vs. privado) y sexo (masculino vs. femenino).

\section{Método}

\section{Participantes}

La selección de los participantes se realizó con apoyo logístico de los estudiantes de la asignatura de psicometría correspondiente a los períodos lectivos del año 2010-2011, de la Escuela de Psicología de la Universidad Metropolitana (UNIMET), ubicada en Caracas, Venezuela. El muestreo utilizado fue de tipo no aleatorio y propositivo (Kerlinger \& Lee, 2002) y como estrategia de maximización de la varianza del puntaje verdadero del Test (Magnuson, 2005) se aseguró que la muestra estuviese balanceada en cuanto a sexo (masculino y femenino) y sector laboral (público y privado).

En total se obtuvo una muestra válida de 233 participantes provenientes del área metropolitana de Caracas. El 18.88\% de la muestra 
poseía al momento del estudio un nivel socioeconómico tipificado como Alto de acuerdo con los estándares de Fundación Centro de Estudios Sobre Crecimiento y Desarrollo de la Población Venezolana (FUNDACREDESA), reseñado tanto por Seijas (2003), como por el Banco Central de Venezuela (BCV, 2007). El 44.64\% poseía un nivel socioeconómico Medio y el restante $36.48 \%$ un nivel socioeconómico Medio-Bajo.

La edad de los participantes se distribuyó entre los 18 y 69 años, con un promedio de 37.01 y un coeficiente de variación media del $32.66 \%$, así como un valor en el coeficiente de asimetría de 0.47 puntos y del coeficiente de curtosis de -0.77 puntos. Esto revela la relativa heterogeneidad de las edades de los participantes (dado el carácter platicúrtico de la distribución) en torno a los 37 años de edad, con una mayor tendencia hacia las edades menores a este valor (debido a la asimetría positiva de la distribución). En términos de desarrollo psicosocial (Papalia \& Olds, $1998)$, puede caracterizarse a la muestra como: adolescentes $(0.43 \%)$, adultos jóvenes (73.39\%) y adultos medios (25.75\%).

\section{Instrumentos}

Maslach Burnout Inventory-General Survey (MBI-GS) (Maslach \& Jackson, 1981)

Se utilizó su tercera edición, validada por Oramas et al. (2007) a partir de la validación española de Moreno-Jiménez, Rodríguez-Carvajal y Escobar (2001). La MBI-GS evalúa la presencia del Síndrome de Burnout para personal latinoamericano que lleva a cabo trabajos dirigidos al área de servicios. La evaluación se hace a partir de tres dimensiones: Desgaste Emocional, Cinismo y Eficacia Profesional. Esta última dimensión es de carácter positivo, ya que está relacionada con las expectativas que se tienen en relación con la profesión que se desempeña, y se expresa en las creencias del sujeto sobre su capacidad de trabajo, su contribución eficaz en la organización laboral, la realización de cosas que realmente valen la pena y su realización profesional; por ello, 
su valoración dentro de la manifestación del Síndrome es inversa a la de las dimensiones de Desgaste Emocional y Cinismo. Los datos obtenidos de una muestra multiocupacional de 122 sujetos cubanos por parte de Oramas et al. (2007) señalan una adecuada consistencia interna del instrumento entre 0.70 y 0.80 , índices correspondientes con los encontrados en el manual del MBI-GS de 0.73 y 0.89 .

Escala de Bienestar Psicológico (EBP) (Sánchez-Cánovas, 2007)

Fue elaborada por Sánchez-Cánovas (2007) en la Universidad de Valencia de España y validada en Venezuela por Millán y D’Aubeterre (2011). Se trata de un cuestionario de administración individual o colectiva, con formato de respuesta tipo Likert de 5 puntos, que puede ser aplicable a adolescentes y adultos de ambos sexos, entre 17 y 90 ańos de edad. Consta de 65 ítems distribuidos en forma diferencial en las siguientes 4 subescalas: Bienestar Psicológico Subjetivo, Bienestar Material, Bienestar Laboral y Relaciones con la Pareja. Para los fines de este estudio y de acuerdo con lo establecido en el baremo de aplicación del instrumento original, se aplicaron solamente los cuadernillos referidos al Bienestar Psicológico Subjetivo, Bienestar Material y Bienestar Laboral. En cuanto a la confiabilidad en la EBP original se reportan coeficientes de consistencia interna alfa de Cronbach $(\alpha)$ entre 0.941 y 0.886 puntos. En el caso de la validación venezolana, Millán y D’Aubeterre (2011) reportan coeficientes de consistencia interna que oscilan entre los 0.923 y 0.866 puntos para cada una de las dimensiones.

Escala Graffar de Estratificación Social (Méndez-Castellano, 1982)

El método Graffar modificado para Venezuela por Méndez-Castellanos (1982) refiere un índice socioeconómico mediante el uso de puntajes asociados a un conjunto de variables, tales como Profesión del Jefe del Hogar, Nivel de Instrucción de la Madre, Principal Fuente de Ingreso y Condiciones de Alojamiento. De acuerdo con Seijas (2003) y el Banco Central de Venezuela (BCV, 2007), la clasificación mediante 
el método Graffar determina 5 estratos a saber: Alto (Nivel I), Medio (Nivel II), Medio Bajo (Nivel III), Obrero (Nivel IV) y Marginal (Nivel V). La confiabilidad del instrumento fue realizada por Contasti (1975) y Laxage, Noguera y Méndez (1986); estos últimos utilizaron el método de consistencia interna por correlación inter-ítems obteniendo coeficientes que varían entre los 0.62 y 0.75 puntos.

Su validación se realizó de acuerdo con Mendoza (2007) en diferentes instancias: a) por un lado, se estudió la estructura unifactorial utilizando el análisis de componentes principales; b) se estudió la estabilidad de la segmentación en cinco clases sociales a partir del análisis de una muestra original de 3850 personas y c) se correlacionaron diversas variables antropométricas y clínicas realizadas en múltiples estudios del Proyecto Venezuela, elaborado por la FUNDACREDESA (como se cita en Mendoza, 2007).

Así mismo, López y Landaeta-Jiménez (2003), señalan que estos estudios no se limitaron al análisis transversal de la escala, sino que continuaron con diseños longitudinales dentro del área metropolitana de Caracas. Finalmente, Aguilera (2009) destaca que en la actualidad la FUNDACREDESA tiene el requerimiento de la evaluación psicométrica de este método Graffar a fin de establecer una nueva actualización del indicador.

\section{Procedimiento}

Se conformó un cuadernillo de aplicación con los instrumentos antes señalados y una carta de presentación explicativa del propósito del estudio y su utilidad como información a los participantes. Se contó con el apoyo de los estudiantes de la Escuela de Psicología de la UNIMET en el levantamiento de información, como parte de los lineamientos de la cátedra para la estandarización de pruebas a la realidad nacional y cumpliendo con el modelo de aprendizaje por proyectos que establece el modelo educativo Diseño Instruccional de la Universidad Metropolitana (DIUM). 
La información recolectada se analizó con el paquete estadístico PASW Statistics 18 (antiguo SPSS), el Microsoft Excel 2007 y el SPAD 5.5. Se realizó el análisis descriptivo de la muestra, el análisis de confiabilidad y el análisis de ítems, para luego proceder a explorar los indicadores que permitieran valorar la validez de constructo del instrumento, por medio del Análisis Factorial de Componentes Principales con Rotación Varimax, con 3 factores predefinidos, dado el carácter confirmatorio $^{3}$ del estudio. Se llevó igualmente a cabo la confirmación de los supuestos de normalidad, independencia y suficiencia que todo instrumento psicométrico debe poseer. Finalmente se analizó la validez divergente de la MBI-GS con respecto a la EBP.

\section{Resultados}

Los resultados obtenidos en el análisis de los ítems revelan que, si bien existe cierta tendencia entre los ítems a agrupar la respuesta promedio en un lado del rango que compone la variable (por ejemplo, los ítems 11,15 y 16 tienen un promedio entre 1 y 2 puntos, en un rango que abarca desde 1 hasta 7 puntos), los mismos poseen una alta heterogeneidad de las respuestas en torno a la media del ítem y, a excepción del ítem 6 , todos poseen un valor aceptable ( $\geq .30$ puntos) en su capacidad discriminativa y su aporte a la comunalidad del instrumento.

3 No confundir con Análisis Factorial Confirmatorio, el cual supone el uso de modelos de parcialización de las correlaciones. 


\section{Tabla 1}

Análisis de items MBI-GS: validación venezolana

\begin{tabular}{ccccccc}
\hline Item & $\begin{array}{c}\text { Media } \\
\text { item } \\
(C=1-7)\end{array}$ & C.V..$_{\text {Media }}$ & $\begin{array}{c}\text { Capacidad } \\
\text { iscriminativa } \\
\left(r_{\text {item-test }}\right)\end{array}$ & $\begin{array}{c}\alpha^{1} \text { sise } \\
\text { elimina } \\
\text { el item }\end{array}$ & $\begin{array}{c}\text { Diferencia } \\
\left(\alpha-\alpha_{\text {-item }}\right)\end{array}$ & $\begin{array}{c}\text { Comuna- } \\
\text { lidades: } \\
\text { Extracción }\end{array}$ \\
\hline 1 & 3.06 & $53 \%$ & .35 & .83 & 0.004 & .59 \\
2 & 2.03 & $68 \%$ & .42 & .83 & 0.007 & .59 \\
3 & 2.41 & $66 \%$ & .62 & .82 & 0.019 & .62 \\
4 & 3.24 & $58 \%$ & .37 & .83 & 0.004 & .62 \\
5 & 2.71 & $63 \%$ & .36 & .83 & 0.004 & .53 \\
6 & 4.30 & $42 \%$ & .22 & .84 & -0.005 & .37 \\
7 & 2.21 & $73 \%$ & .38 & .83 & 0.005 & .39 \\
8 & 3.30 & $57 \%$ & .43 & .83 & 0.008 & .50 \\
9 & 2.67 & $66 \%$ & .64 & .82 & 0.021 & .53 \\
10 & 2.11 & $74 \%$ & .48 & .83 & 0.011 & .64 \\
11 & 1.97 & $71 \%$ & .68 & .82 & 0.020 & .59 \\
12 & 2.21 & $70 \%$ & .70 & .81 & 0.023 & .61 \\
13 & 3.27 & $64 \%$ & .36 & .84 & 0.001 & .46 \\
14 & 2.32 & $73 \%$ & .42 & .83 & 0.008 & .44 \\
15 & 1.82 & $73 \%$ & .45 & .83 & 0.009 & .61 \\
16 & 1.88 & $70 \%$ & .49 & .83 & 0.010 & .71 \\
\hline & & & & & &
\end{tabular}

En el caso del ítem 6, si bien posee un menor aporte a la consistencia interna de la prueba, su inclusión supone una muy poca disminución de dicha consistencia interna $(-0.005)$ y en ningún caso supone su diminución a niveles inaceptables de confiabilidad (el alfa obtenido para la prueba en general, posterior a la recodificación de los ítems de eficacia profesional, como ítems de ineficacia profesional es de 0.84). Puede estarse en presencia de la paradoja de la teoría clásica de los test (Muñiz, 1998), la cual supondría la posibilidad de sacrificar 
cierto porcentaje de confiabilidad de la prueba final (en específico el $0.006 \%$ ) en aras de maximizar la validez del cuestionario, lo cual se confirmará analizando: a) el aporte del ítem 6 a la correspondencia entre la estructura factorial con respecto a la teórica prescrita en la tabla de especificaciones del cuestionario, b) el porcentaje de explicación de dicho ítem 6 con respecto a tal estructura factorial obtenida, c) el aporte del ítem 6 al logro de la normalidad de la prueba, y d) el aporte del ítem 6 a la capacidad predictiva del criterio de Bienestar Psicológico.

El análisis de consistencia interna revela la adecuación del instrumento a los estándares referidos por Prieto y Muñiz (2000) con respecto al valor del coeficiente de consistencia interna $(\alpha \geq .70)$, así como también un mayor puntaje en los mismos si se compara con el estudio original de Maslach y Jackson (1981) y el estudio de Oramas et al. (2007); a excepción del componente de Agotamiento Emocional, que en comparación con este último estudio es levemente inferior. Así mismo, el mayor puntaje reseńado por el estudio de Moreno-Jiménez et al. (2001) puede deberse a: a) el uso de una muestra de tan solo 114 participantes, la cual es catalogada como pobre (Peña, 2009) o pequeña (Comisión de los Tests del Colegio Oficial de Psicólogos de España, como se cita en Prieto \& Muñiz, 2000), sin contar lo ya señalado para el criterio de potencia estadística del análisis factorial; b) la homogeneidad de la muestra, puesto que todos los participantes son empleados administrativos, en su mayoría con una jornada compacta de trabajo (89.5\%), mujeres $(79.8 \%)$, de edad media $\left(X=42 ; C . V_{X_{X}}=19.33 \%\right)$, con pareja estable $(68.4 \%)$ y con niveles de estudio medio $(64.9 \%)$, lo cual, como ya se señaló anteriormente, es contraproducente en cualquier estudio desarrollado a partir de análisis de varianzas conjuntas o correlaciones, en especial cuando se utiliza el análisis factorial. 


\section{Tabla 2}

Consistencia interna MBI-GS: validación venezolana

\begin{tabular}{lccccc}
\hline $\begin{array}{l}\text { Dimensiones } \\
\text { de medida }\end{array}$ & $\begin{array}{c}\text { Millán \& } \\
\text { D'Aubeterre } \\
\text { (2011) }\end{array}$ & $\begin{array}{c}\text { Oramas } \\
\text { et al. } \\
\text { (2007) }\end{array}$ & $\begin{array}{c}\text { Maslach } \\
\text { \&ackson } \\
\text { (1981) }\end{array}$ & $\begin{array}{c}\text { Morenónez et al. } \\
\text { (2001) }\end{array}$ & $\begin{array}{c}N^{\circ} \\
\text { items }\end{array}$ \\
\hline Total de la Prueba & 0.84 & 0.77 & No reporta & No reporta & 16 \\
Cinismo & 0.83 & 0.78 & 0.68 & 0.84 & 5 \\
Agotamiento emocional & 0.70 & 0.70 & 0.73 & 0.89 & 5 \\
Baja eficacia profesional & 0.81 & 0.80 & 0.78 & 0.85 & 6 \\
\hline
\end{tabular}

La validación de constructo se realiza, por un lado, a partir de la coincidencia entre la distribución teórica de los ítems (tabla de especificaciones del test) y la distribución empírica de los mismos a partir de la intercorrelación entre ellos (Análisis Factorial) y, por el otro, a partir de la correlación moderada y significativa entre el puntaje global del test y otro test ya validado en la población de estudio, con el cual se posea un vínculo semántico y sintáctico (Arnau, 1979). En este caso por tratarse de Bienestar Psicológico la hipótesis es de divergencia y, por lo tanto, la direccionalidad esperada en el coeficiente de correlación es negativo o inverso; es decir, a mayor puntaje de Burnout, menor puntaje de Bienestar Psicológico.

En el caso del Análisis Factorial Exploratorio prefijado a 3 factores, se confirmaron los supuestos de suficiencia de la estructura factorial obtenida e independencia entre los factores, ya que por un lado la prueba de adecuación muestral de Kaiser-Meyer y Olkin $(\mathrm{KMO}=0.85)$ tuvo un puntaje superior al criterio de 0.50 puntos (Pardo \& Ruiz, 2005), lo que puede interpretarse inclusive como meritorio (Peńa, 2009). El $p_{\text {valor }}$ de la Prueba de Esfericidad de Bartlett (Sig. $=0.000$ ), fue menor al criterio de 0.05 puntos, ya que se consideró una confianza de $95 \%$ $(1-\alpha)$. Las correlaciones entre factores (Tabla 3) no fueron ni significativas ni cercanas a 0 , a diferencia de lo que sucede con la suma simple de los ítems, lo cual incumple con el supuesto de independencia. 


\section{Tabla 3}

Intercorrelaciones entre los puntajes obtenidos por el método de regresión al factor y la suma simple de items

\begin{tabular}{lccccc}
\hline & & \multicolumn{2}{c}{ Suma Simple } & \multicolumn{2}{c}{ Puntaje Factorial } \\
\cline { 3 - 6 } & & Eficacia & Agotamiento & Eficacia & Agotamiento \\
\hline \multirow{2}{*}{ Cinismo } & $\mathrm{r}$ & -.43 & .62 & -.06 & .01 \\
& Sig. (bilateral) & $.000^{* *}$ & $.000^{* *}$ & .39 & .85 \\
\hline
\end{tabular}

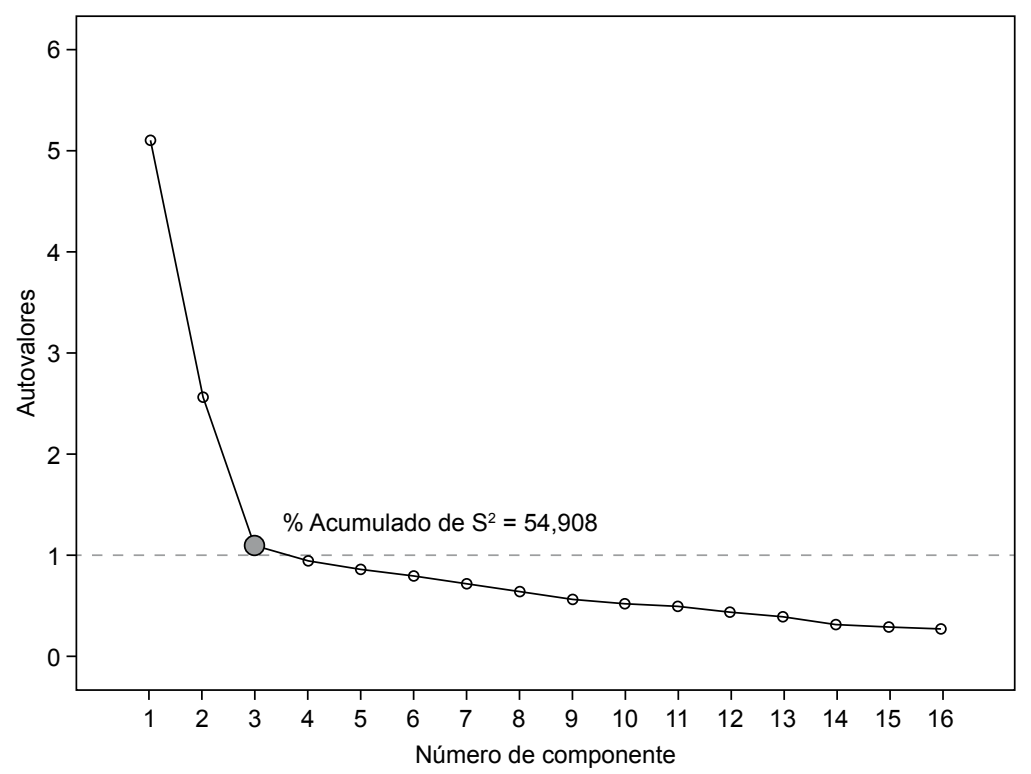

Figura 1. Gráfico de sedimentación del AFE.

El gráfico de sedimentación (Figura 1) revela que si bien la corrida del Análisis Factorial Exploratorio fue prefijada a 3 factores dado el carácter confirmatorio del estudio, de haberlo dejado totalmente libre hubiese aparecido igualmente el modelo de 3 factores ya que es hasta este factor que se mantienen los autovalores superiores a 1 (límite de la 
línea pespunteada), cumpliendo con el criterio de Gutman-Kaiser para selección del número de factores dentro del modelo factorial (Morales, 2008). Así mismo, es con el modelo trifactorial que se cumple con el criterio de Scree Test de Cattell (Peña, 2009), también conocido como el criterio del codo (Hurtado, 2010), puesto que es en este punto donde se encuentra la inflexión o asíntota en la acumulación de autovalores obtenidos. Finalmente, el porcentaje de varianza explicada por el modelo de 3 factores (ver Tabla 4) supera el límite de 50\% señalado por Morales (2008).

Todo lo anterior confirma entonces la presencia de un modelo trifactorial, tal como lo refleja el modelo teórico original del MBI-GS. Ahora, para seguir con la validación de constructo sobre la base de la información interna del instrumento, se verificó que la distribución de los ítems entre sí coincidiera con lo establecido por la tabla de especificaciones del instrumento, a partir de la inspección del gráfico que representa la ubicación espacial de cada ítem y de cada factor del modelo representado como un vector, así como también a partir de su ubicación en la matriz de componentes rotados obtenidos por medio del Análisis Factorial Exploratorio con Rotación Varimax.

En tal sentido, tanto en la Figura 2 como en la Tabla 4 se observa que salvo el ítem 1 (vector pespunteado), el resto de los ítems mantienen la ubicación empírica esperada a partir del constructo teórico, validando el mismo. En el caso de este ítem, desde el punto de vista teórico, pertenece a la dimensión Agotamiento Emocional, sin embargo, su ubicación relativa a partir de las respuestas de los participantes de la muestra lo ubica dentro del cuadrante que representa a los ítems de Cinismo.

Así mismo, resalta que el ítem 13 (marcado con asterisco) coincide con la ubicación teórica del instrumento original de Maslach y Jackson (1981), así como la validación española del mismo hecha por Moreno-Jiménez et al. (2001). El ítem 12 coincide con la validación cubana de Oramas et al. (2007). 
Facteur 1

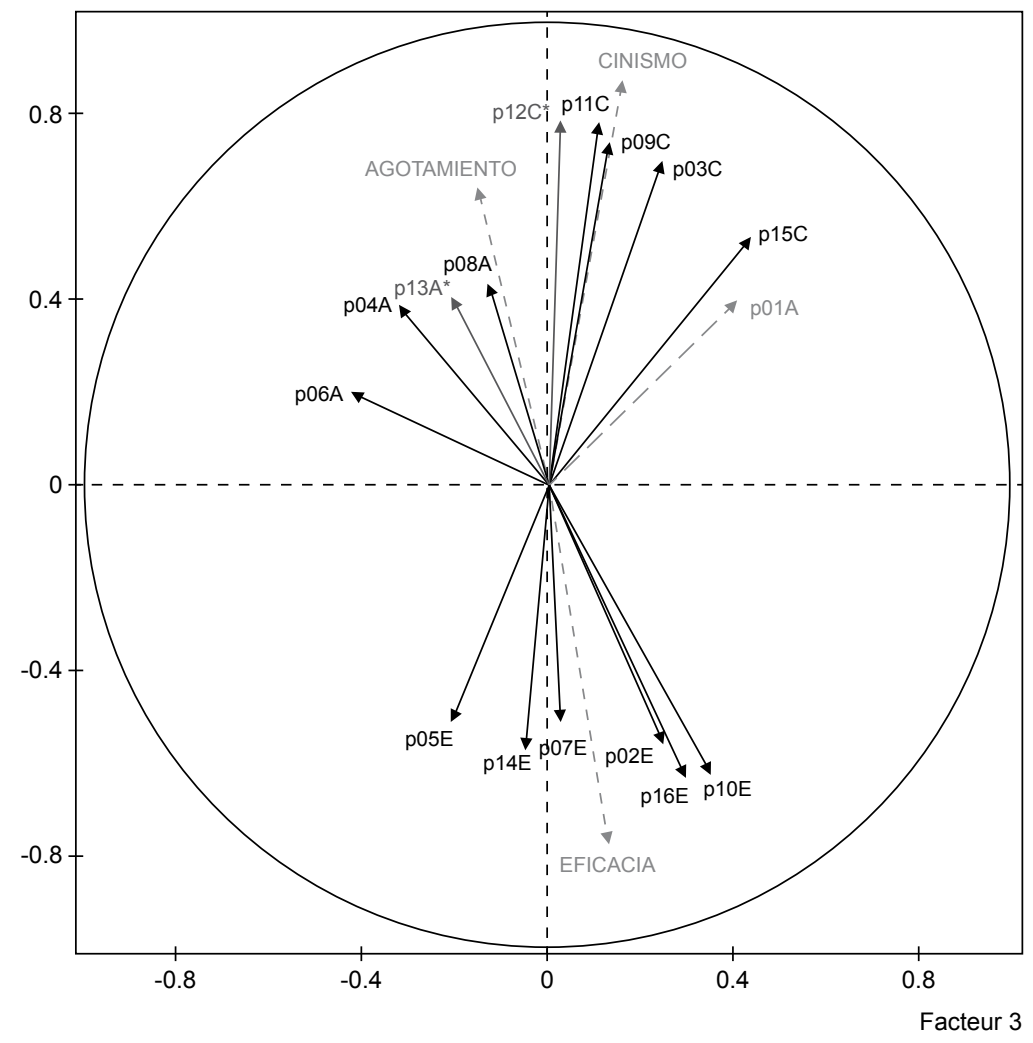

Figura 2. Gráfico de distribución vectorial - ítem x factor.

Los resultados de los indicadores de los supuestos de independencia, suficiencia y ubicación relativa de los ítems, revelan que la posibilidad de eliminar al ítem 6 del instrumento por falta de capacidad discriminativa, queda anulada puesto que en el resto de indicadores internos del instrumento este se comporta dentro de los parámetros esperados. No es así en el caso del ítem 1 que internamente debía comportarse como un ítem perteneciente a la dimensión de Agotamiento Emocional y en realidad se comporta como un ítem de Cinismo, por lo 
que se recomienda su anulación al analizarse el Síndrome del Burnout en la población venezolana, para asegurar la debida consistencia semántica o de constructo del instrumento.

\section{Tabla 4}

Matriz de componentes rotados: items de eficacia

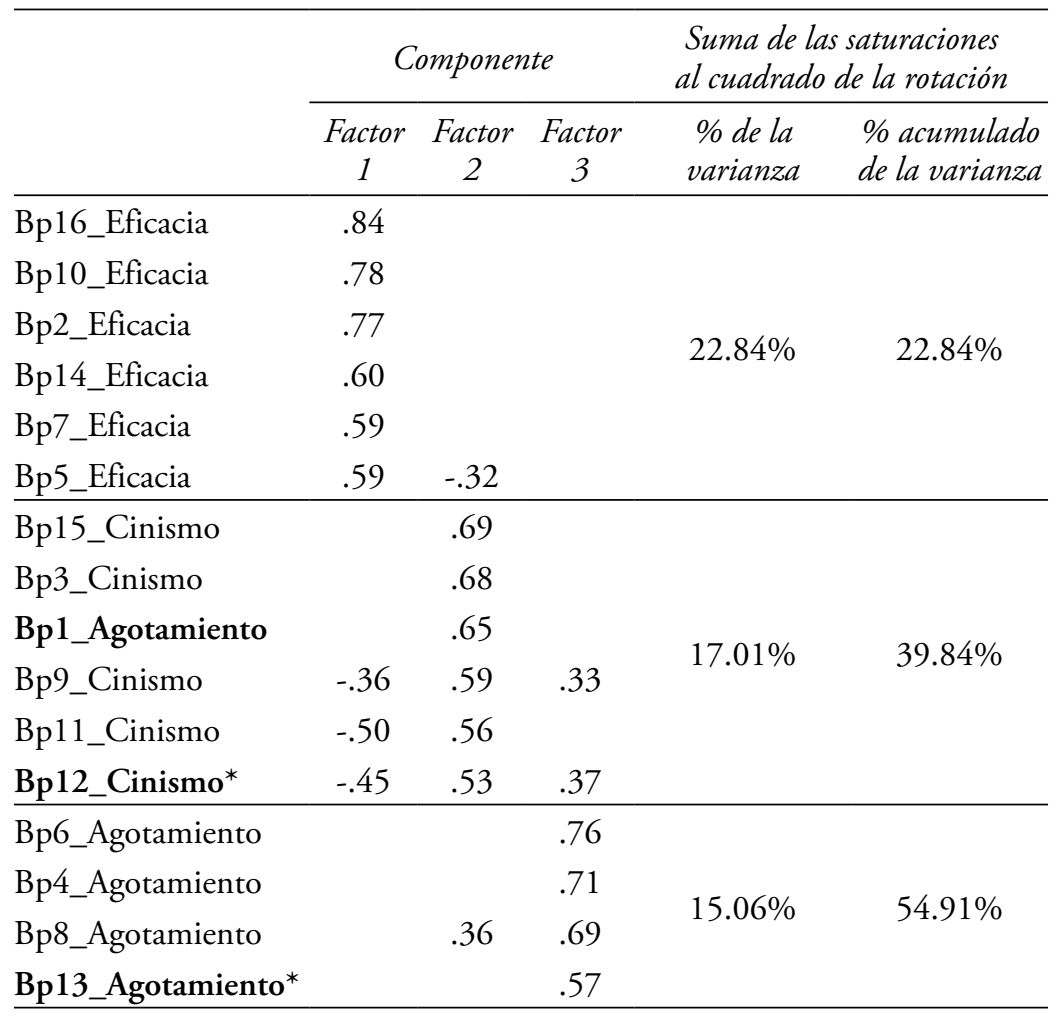

La inspección gráfica de la Figura 2 lleva también a la reflexión en torno a si la denominación del Factor 1 debería ser: Eficacia Profesional, Baja Eficacia Profesional o Ineficacia Profesional, la cual ha estado presente en la historia misma del desarrollo del instrumento, tal como lo señala Shirom (2009), pero que en todo caso representa 
las autoevaluaciones de la baja autoeficacia, carencia de logros, falta de productividad e incompetencia. En tal sentido, la ubicación vectorial de los ítems en un cuadrante diferente al de Cinismo y Agotamiento hace que no sea pertinente pensar en términos negativos con respecto a dicho factor, puesto que dicha proyección supondría su anulación ya que se solaparía con los mencionados ítems de Cinismo y Agotamiento. Con respecto al supuesto de normalidad (Tabla 5), se observa que salvo en el factor de agotamiento emocional, en la muestra de participantes evaluados no se cumple el mismo ya que el $\mathrm{P}_{\text {valor }}$ (Sig.) obtenido es inferior al criterio de .05 puntos, tanto para el puntaje de cada factor como a partir del puntaje factorial por medio de la ecuación de regresión pertinente según los coeficientes obtenidos (ver Tabla 5).

\section{Tabla 5}

Matriz de coeficientes para el cálculo de las puntuaciones en las componentes

\begin{tabular}{lccc}
\hline & $\begin{array}{c}\text { Factor 1 } \\
\text { Eficacia }\end{array}$ & $\begin{array}{c}\text { Factor 2 } \\
\text { Cinismo }\end{array}$ & $\begin{array}{c}\text { Factor 3 } \\
\text { Agotamiento }\end{array}$ \\
\hline Bp2_Eficacia & .28 & .15 & -.03 \\
Bp3_Cinismo & .10 & .36 & -.03 \\
Bp4_Agotamiento & -.04 & -.15 & .36 \\
Bp5_Eficacia & .14 & -.11 & .18 \\
Bp6_Agotamiento & -.01 & -.19 & .40 \\
Bp7_Eficacia & .13 & -.08 & .10 \\
Bp8_Agotamiento & .08 & .07 & .26 \\
Bp9_Cinismo & .00 & .22 & .04 \\
Bp10_Eficacia & .30 & .22 & -.12 \\
Bp11_Cinismo & -.03 & .23 & -.02 \\
Bp12_Cinismo & -.08 & .09 & .12 \\
Bp13_Agotamiento & .00 & -.01 & .23 \\
Bp14_Eficacia & .17 & -.03 & .05 \\
Bp15_Cinismo & .17 & .48 & -.18 \\
Bp16_Eficacia & .31 & .19 & -.06 \\
\hline
\end{tabular}




\section{Tabla 6}

Indicadores del supuesto de normalidad del puntaje total del MBI-GS

\begin{tabular}{|c|c|c|c|c|c|}
\hline & & & Estadístico & $g l$ & Sig. \\
\hline \multirow{6}{*}{$\begin{array}{l}\text { Puntaje por } \\
\text { dimensión } \\
\text { o factor }\end{array}$} & \multirow{3}{*}{$\begin{array}{l}\text { Suma } \\
\text { simple }\end{array}$} & Cinismo & .11 & 233 & .000 \\
\hline & & Eficacia profesional & .15 & 233 & .000 \\
\hline & & $\begin{array}{l}\text { Agotamiento o } \\
\text { Desgaste emocional }\end{array}$ & .07 & 233 & .005 \\
\hline & \multirow{3}{*}{$\begin{array}{l}\text { Puntaje } \\
\text { factorial }\end{array}$} & Factor 1: Eficacia & .13 & 233 & .000 \\
\hline & & Factor 2: Cinismo & .12 & 233 & .000 \\
\hline & & $\begin{array}{l}\text { Factor 3: } \\
\text { Agotamiento }\end{array}$ & .05 & 233 & $.200^{* *}$ \\
\hline \multirow{4}{*}{$\begin{array}{l}\text { Puntaje } \\
\text { final }\end{array}$} & \multirow{2}{*}{$\begin{array}{l}\text { Sin } \\
\text { normalizar }\end{array}$} & $\begin{array}{l}\text { Total Suma Ítems } \\
\text { (Ítems de Eficacia } \\
\text { Rotados) }\end{array}$ & .09 & 233 & .000 \\
\hline & & $\begin{array}{l}\text { Total Suma Factores } \\
\text { (Ítems de Eficacia } \\
\text { Rotados) }\end{array}$ & .08 & 233 & .002 \\
\hline & \multirow[b]{2}{*}{ Normalizados } & $\begin{array}{l}\text { Log } 10 \text { Total Suma } \\
\text { Factores }\end{array}$ & .06 & 233 & $.061^{* *}$ \\
\hline & & $\begin{array}{l}\text { Log Total Factores: } \\
\text { Cinismo + } \\
\text { Agotamiento - } \\
\text { Eficacia }\end{array}$ & .06 & 233 & $.061^{* *}$ \\
\hline
\end{tabular}

Se observó que también es factible la normalización de estos puntajes aplicando como constante a cada puntaje su Logaritmo base 10, tanto para el cálculo del puntaje total por medio de la rotación previa de los ítems de Eficacia Profesional, como en el caso en que se suma primero el valor del Factor Cinismo y Agotamiento Emocional, para luego restar el valor del Factor de Eficacia Profesional (ver Tabla 6 y Figura 3). 

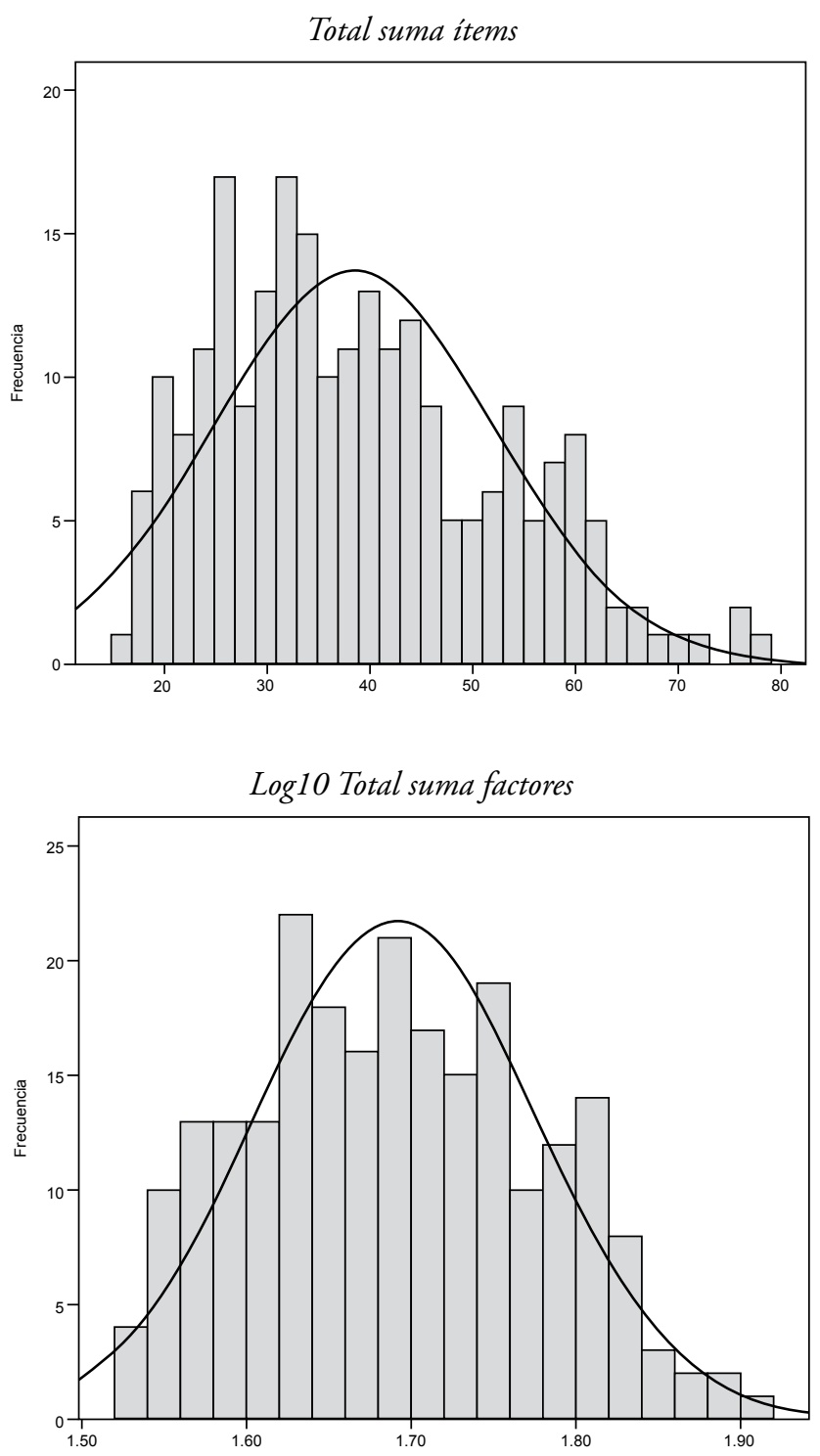

Figura 3. Histogramas del puntaje del MBI-GS calculado como suma simple de los ítems y normalizado a partir del $\mathrm{LOG}_{10}$. 

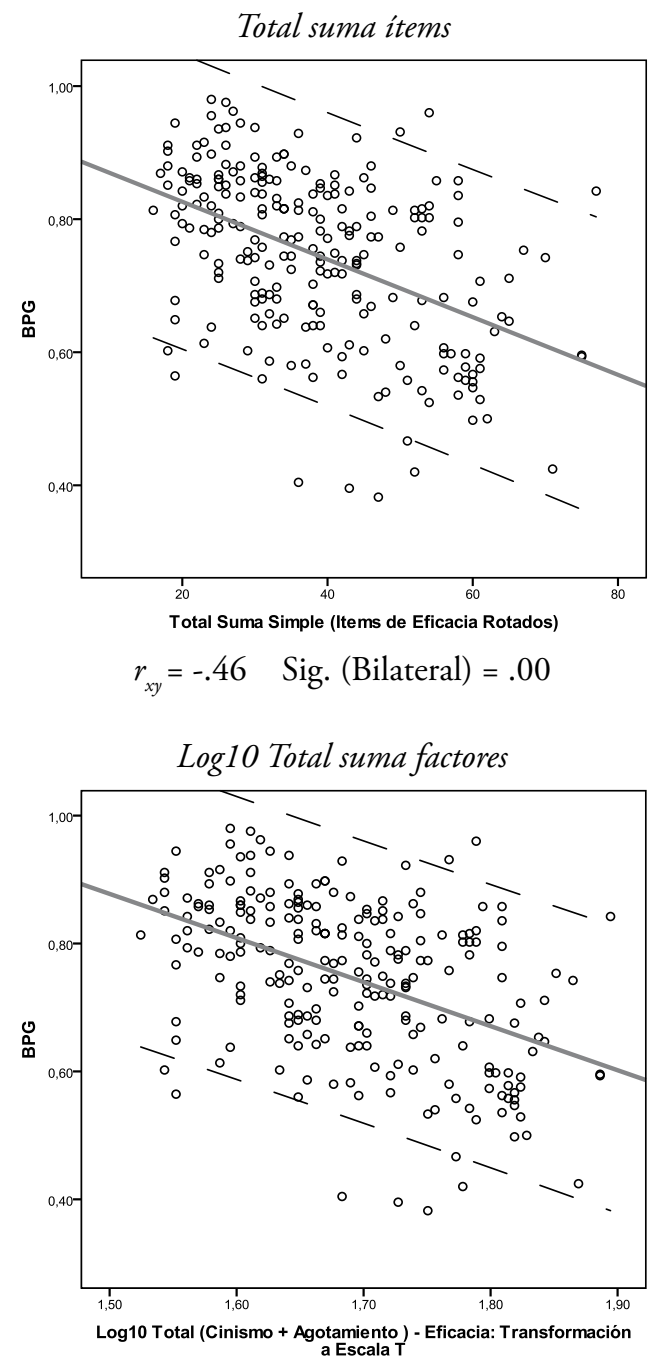

$$
r_{x y}=-.46 \text { Sig. }(\text { Bilateral })=.00
$$

Figura 4. Dispersigramas entre el puntaje del MBI-GS calculado como suma simple de los ítems y normalizado a partir del LOG ${ }_{10}$, con respecto a la EBP. 
Esta normalización permite satisfacer el supuesto de normalidad estadística que requieren tanto los test psicométricos, como algunos estadísticos paramétricos, sin que se sacrifique la información obtenida ya que se mantiene la posición relativa de cada participante en relación con el resto de sujetos que pertenecen a la muestra (Magnuson, 2005). En cuanto a la validez divergente del MBI-GS, se observó que la correlación del MBI-GS con el puntaje general de Bienestar Psicológico (ver Figura 4) cumplió con los siguientes criterios: mayor a 0.30, significativo y con direccionalidad inversa, lo cual supone que a mayores puntajes en el MBI-GS, menores puntajes en el EBP se tenderá a poseer.

\section{Discusión}

Los resultados indican que en la muestra estudiada la versión latinoamericana del MBI-GS, desarrollada por Oramas et al. (2007) es satisfactoria si se prescinde del ítem 1: "Me siento emocionalmente agotado(a) por mi trabajo", debido a que el mismo posee una carga factorial que lo vincula con los ítems de la dimensión de Cinismo y no con la dimensión de Agotamiento Emocional que establece el manual original del instrumento. Así mismo, resaltan los casos de los ítems 12 y 13, cuyas afirmaciones son: "Me siento 'quemado(a)', 'hastiado(a)' de mi trabajo" y "Sólo quiero hacer lo mío y que no me molesten", respectivamente, ya que en el caso del presente estudio se mantiene la posición sugerida por Oramas et al. (2007), dentro de la dimensión de Cinismo, quizás por el ajuste hecho a la frase, que originalmente señalaba: "me siento quemado por mi trabajo", por lo que la incorporación del segmento: "hastiado por mi trabajo", hace que se incluya una noción de aversión y rechazo compatible con el cinismo más que con el desgaste emocional, como lo señala la versión de Moreno-Jiménez et al. (2001), sobre la cual versa este instrumento.

En cambio, en el caso del ítem 13, se mantiene la disposición del ítem dentro de la dimensión de Agotamiento, lo cual resulta lógico dado que su contenido no fue sometido a cambio alguno por parte de 
Oramas et al. (2007). La diferencia quizás dependa, tal como se señaló anteriormente, del tamaño y la homogeneidad de la muestra, ya que todos los participantes se encontraban en la sala de Clínica Ocupacional del Instituto Nacional de Salud de los Trabajadores (INSAT) y posiblemente provengan de una misma categoría profesional, cuya visión sobre el trabajo en equipo sea igualmente particular, o finalmente por las diferencias socio económicas y culturales, propias del sistema económico particular de la isla de Cuba, que la distinguirían del resto de las muestras estudiadas. Por ello se recomienda mayores estudios al respecto de tipo comparativo, y salvando el tema del tamaño y la homogeneidad de la muestra.

Con respecto a la confiabilidad, se observa que los valores encontrados son adecuados aunque levemente diferentes a lo que, de acuerdo con Oramas et al. (2007), ha sido la tendencia tradicional en el estudio del MBI-GS. En este caso la dimensión de Cinismo no resultó ser la menos favorecida de las tres dimensiones sino, por el contrario, fue la de mayor puntaje en el coeficiente de consistencia interna alfa de Cronbach. Así mismo, Agotamiento Emocional tampoco resultó ser, en comparación con la tradición, la dimensión con mayor consistencia interna, sino más bien la menor, aunque siempre dentro de los parámetros establecidos como satisfactorios.

Como valor agregado del estudio se explora la existencia de la normalidad en las distribuciones, aspecto que en los estudios previos analizados no fue realizado, pero cuya importancia es capital para asegurar la máxima discriminación interna de los sujetos con respecto al continuo subyacente de capacidad y para asegurar la continuidad métrica de la variable Burnout. En tal sentido se recomienda realizar este tipo de análisis a la hora de validar y, cuando este supuesto no se asegure, se recomienda su normalización al aplicar el $\log _{10}$ sobre cada puntaje obtenido.

Importante también es la ampliación del proceso de validación al uso de indicadores externos al propio test, que en este caso supuso la validación divergente del instrumento. Finalmente, se presentan evidencias empíricas que justifican la denominación de Baja Eficacia Profesional, en contraposición con la denominación de Ineficacia Profesional, 
ya que representan dimensiones diferenciales y suponer una inversión de la direccionalidad del vector significaría la pérdida del factor al solaparse sus ítems dentro del factor de Agotamiento Emocional y Cinismo.

\section{Referencias}

Aguilera, W. (2009). Evaluación del índice Graffar-Méndez Castellano mediante el uso de técnicas psicométricas en el marco del SENACREDH. Revista de la Sociedad Médico Quirúrgica del Hospital de Emergencia Pérez de León, 40(1), 99-101.

Anastasi, A. \& Urbina, S. (1998). Tests psicológicos (7ma. ed.). México D. F.: Prentice Hall.

Arnau, J. (1979). Psicología experimental: un enfoque metodológico. México D. F.: Trillas.

Aron, A. \& Aron, E. (2001). Estadistica para psicología (2da. ed.). México D. F.: Prentice Hall.

Banco Central de Venezuela. (2007). III Encuesta Nacional de Presupuestos Familiares. Caracas: Gerencia de Estadísticas Económicas.

Contasti, M. (1975). Graffar-Méndez Castellano. Estructura y validez. En H. Méndez \& M. C. Méndez (Eds.), Sociedad y estratificación. Método Graffar-Méndez Castellano (pp. 157-174). Caracas: Fundacredesa.

Garcés de los Fayos, E., López, S. \& García, M. (1997). El sindrome de "Burnout" y su evaluación: una revisión de los principales instrumentos de medida. Cartel presentado en el I Congreso de la Asociación Española de Psicología Clínica y Psicopatología, Universidad de Murcia, España.

Gil-Monte, P. (2001). Validez factorial de la adaptación al español del Maslach Burnout Inventory-General Survey. Salud Pública de México, 44(1), 33-40.

Gil-Monte, P. (2005). El sindrome de quemarse por el trabajo (Burnout). Una enfermedad laboral en la sociedad de bienestar. Madrid, España: Pirámide. 
Hair, J., Anderson, R., Tatham, R. \& Black, W. (1999). Análisis multivariante. Madrid, Espańa: Prentice Hall.

Hernández, T., Terán, O., Navarrete, D. \& León, A. (2007). Síndrome de Burnout: una aproximación a su conceptualización, antecedentes, modelos explicativos y de medición. Revista Internacional La Nueva Gestión Organizacional, 3(5), 50-68.

Hurtado, J. (2010). Metodología de la investigación: guí para la comprensión holística de la ciencia (4ta. ed.). Caracas, Venezuela: Quirón.

Kerlinger, F. \& Lee, H. (2002). Investigación del comportamiento: métodos de investigación en ciencias sociales. México D. F.: Mc Graw Hill.

Laxage, G., Noguera, G. \& Méndez, H. (1986). Investigación sobre la consistencia de las variables utilizadas en el método Graffar modificado. Archivos Venezolanos de Puericultura y Pediatría, 49(1), 105-155.

López, M. \& Landaeta-Jiménez, M. (2003). La antropometría en el estudio del crecimiento y desarrollo físico: experiencia venezolana. Revista Española de Nutrición Comunitaria, 9(3), 128-136.

Magnuson, D. (2005). Teoría de los test. México D. F.: Trillas.

Maslach, C. (2009). Comprendiendo el Burnout. Ciencia y Trabajo, 11(32), 37-43.

Maslach, C. \& Jackson, S. (1981). Maslach Burnout Inventory Manual (10ta. ed.). Palo Alto, CA: Consulting Psychologists Press.

Méndez-Castellano, H. (1982). Método Graffar modificado para Venezuela: Manual de procedimientos del área de familia. Caracas, Venezuela: Fundacredesa.

Mendoza, N. (2007). Efectos de un programa social sobre el desarrollo social, estilos de vida y calidad de vida, relacionada con la salud en población rural venezolana: validación transcultural del medida de salud SF-36 en población ruralde Venezuela (Tesis doctoral inédita). Universidad de Alicante, España. Recuperada de http://www.lluisvives.com/ servlet/SirveObras/45705174320147210732679/031084.pdf Millán, A. \& D’Aubeterre, M. E. (2011). Validación de la Escala de Bienestar Psicológico en una muestra multiocupacional venezolana. Revista CES Psicología, 4(2), 52-71. 
Morales, P. (2008). El análisis factorial en la construcción e interpretación de tests, escalas y cuestionarios. Manuscrito inédito, Departamento de Metodología y Evaluación, Universidad Pontificia Comillas, Madrid, España.

Moreno-Jiménez, B., Rodríguez-Carvajal, R. \& Escobar, E. (2001). La evaluación del burnout profesional: factorización del MBI-GS. Un análisis preliminar. Ansiedad y Estrés, 7(1), 69-78.

Muñiz, J. (1998). Teoría clásica de los test. Madrid, España: Pirámide.

Oramas, A., González, A. \& Vergara, A. (2007). El desgaste profesional: evaluación y factorialización del MBI-GS. Revista Cubana de Salud y Trabajo, 8(1), 37-45.

Papalia, D. \& Olds, S. (1998). Psicología. Madrid, España: Mc Graw Hill.

Pardo, A. \& Ruiz, M. (2005). Spss 13: guia para el análisis de datos. Madrid, España: Mc Graw Hill.

Peña, G. (2009). Estadistica inferencial: una introducción para las ciencias del comportamiento. Caracas, Venezuela: Universidad Católica Andrés Bello.

Pines, A. (1987). Marriage burnout: A new conceptual framework for working with couples. Psychotherapy in Private Practice, 5(1), 31-43.

Prieto, G. \& Muñiz, J. (2000). Un modelo para evaluar la calidad de los test utilizados en España. Papeles del Psicólogo, 77(1), 65-75.

Sánchez-Cánovas, J. (2007). Manual Escala de Bienestar Psicológico (2da. ed.). Madrid, España: TEA.

Seijas, F. (2003). Investigación electoral: encuestas electorales (2da. ed.). Caracas, Venezuela: Ediciones del Rectorado - Universidad Central de Venezuela.

Shirom, A. (2009). Acerca de la validez del constructo, predictores y consecuencias del burnout en el lugar de trabajo. Ciencia y Trabajo, 11(32), 44-54.

Recibido: 31 de julio, 2011 Aceptado: 30 de octubre, 2011 